\title{
Relevance of surface roughness to tungsten sputtering and carbon implantation
}

Ivan Bizyukov

Kharkiv National University, Faculty of Physics and Technologies, 31 Kurchatov Ave., Kharkiv 61108, Ukraine

Karl Krieger

Max-Planck-Institut für Plasmaphysik, EURATOM Association, Boltzmannstr. 2, 85748 Garching, Germany

Nikolay Azarenkov

Kharkiv National University, Faculty of Physics and Technologies, 31 Kurchatov Ave., Kharkiv 61108, Ukraine

Udo v. Toussaint

Max-Planck-Institut für Plasmaphysik, EURATOM Association, Boltzmannstr. 2, 85748 Garching, Germany

\begin{abstract}
Tungsten sputtering and carbon layer growth by carbon ion bombardment has been investigated by experiments with thin W layers. Preparation of tungsten layers by magnetron deposition allows one to control the surface roughness by choosing appropriate substrates. The fluence dependent elemental composition of the bombarded surface has been studied insitu with ion beam analysis allowing separate measurements of the areal densities of tungsten and carbon atoms. In contrast to weight-loss measurements, this approach is much less affected by non-uniformities of the incident flux and therefore allows one to determine the dependency of the principal physics processes on input parameters with much higher
\end{abstract}


accuracy. After bombardment, ex-situ scanning electron microscopy has been used for the qualitative understanding of the evolution of the surface topography with increasing fluence. The experiments clearly show the influence of surface roughness, leading to increased tungsten sputter yields and strongly reduced carbon implantation rates. Comparison of the experimental results with simulations by the Monte-Carlo code TRIDYN has verified the validity of the kinematic description for the interaction of carbon ions with mixed carbontungsten surfaces. At the same time, chemical effects, like carbide formation, can be neglected in comparison to the contribution of surface roughness to the dynamics of sputtering and implantation.

Keywords: roughness, ion bombardment, carbon implantation, tungsten sputtering 


\section{Introduction}

In the design of International Tokamak Experimental Reactor (ITER), tungsten (W) will be used for plasma-facing components (PFCs), which are exposed to high particle and heat fluxes ([1], [2], [3]) and complete coverage of the first wall with tungsten is envisaged in future demo reactor devices. Analysis of W PFCs in ASDEX Upgrade has shown that the main erosion mechanism for tungsten is sputtering by multiply charged low-Z impurities, which can be accelerated in the sheath potential to energies of up to several $\mathrm{keV}$ [4]. As the main wall and divertor plates of ITER consist of beryllium and carbon (C) respectively with predicted plasma concentrations in the $1 \%$ range, a similar erosion mechanism can be expected for the W surfaces in ITER.

A number of experiments on bombardment of $\mathrm{W}$ surfaces with $\mathrm{C}$ ions under wellcontrolled laboratory conditions were performed using in-situ weight-loss measurements. In particular the dependence of the sputtering dynamics on the energy and angle of incidence of $\mathrm{C}$ ions has been studied [5]. It turned out that ion bombardment at angles $<40^{\circ}$ leads to $\mathrm{C}$ implantation, which is initially accompanied by W sputtering and turns at a certain incident fluence into continuous growth of a $\mathrm{C}$ layer. The growth rate of the $\mathrm{C}$ layer, detected by the corresponding increase of the sample weight, was in good agreement with simulations by the Monte-Carlo code TRIDYN [6]. However, the evolution of the initial weight loss, which corresponds to sputtering of $\mathrm{W}$ and the transition from sputtering to $\mathrm{C}$ layer growth, could be only qualitatively reproduced. The strongest disagreement was found for the weight loss of the sample in the phase where a surface layer of mixed $\mathrm{C}$ and $\mathrm{W}$ atoms is bombarded with $\mathrm{C}$ projectiles. Continuous sputtering of $\mathrm{W}$ at inclined angles of incidence, where steady state is reached at much lower fluence, was reproduced much better. Subsequent studies, which included data on the bombardment of $\mathrm{W}$ with $\mathrm{C}$ ions at room temperature $[7,8]$, have shown that the observed differences between the simulated and experimentally measured dynamics 
of the surface modification is clearly outside the experimental errors.

The described deviations are assumed to originate mainly from inaccuracies of the experimental approach of weight loss measurements and/or by incorrect simplifications in modeling. The most significant experimental inaccuracies are unknown surface conditions, like roughness and cleanness of the bombarded surface, which may also change with increasing incident fluence and lead to an increased sputter yield. Furthermore, a non-uniform particle flux distribution will significantly affect the weight change dynamics, because edge regions of the irradiation spot still dominated by sputtering contribute to a decrease of the total sample weight, while the center of the irradiation spot with higher local fluence already forms a growing $\mathrm{C}$ layer with corresponding weight increase. Apart from these experimental uncertainties, simulations can also significantly deviate from the actual behavior because properties of the surface layer with mixed materials, particularly density, can strongly deviate from the usually assumed linear superposition of the corresponding values of the pure materials

Accurate modeling of the interaction of $\mathrm{W}$ surfaces with $\mathrm{C}$ ions is important, particularly with respect to the study of even more complex multi-species bombardment, like simultaneous incidence of $\mathrm{C}$ and $\mathrm{D}$ ions. This leads to additional processes like chemical interactions between the implanted projectiles and diffusion of $\mathrm{C}$ at high temperatures, which may significantly alter sputtering and implantation dynamics [9].

Consequently, in order to quantitatively study the contribution of these additional processes, the sources of disagreement between experimental results and simulations of pure C bombardment of W surfaces have to be identified and eliminated. Control over the initial surface roughness can be achieved by replacing polished bulk poly-crystalline $\mathrm{W}$ as target by thin layers deposited on surfaces with different roughness, like atomically smooth Si wafers versus polished pyrolitic graphite. Even more important is the replacement of weight-loss 
measurements by in-situ ion beam analysis (IBA) of the surface because this ultimately eliminates the errors due to non-uniformities of the incident ion beam profile. The results presented in this study allow for the first time extraction of qualitatively new information on ion-surface interaction in comparison to weight-loss measurements. Finally, the comparison of the obtained experimental data with simulations by the Monte-Carlo code TRIDYN is discussed.

\section{Experimental}

The experiments were performed on a recently commissioned dual ion beam setup [10]. The solid state sputter ion source of this system was used to produce a beam of massseparated $12 \mathrm{keV} \mathrm{C}_{2}^{-}$ions with a resulting energy of $6 \mathrm{keV}$ per incident $\mathrm{C}$ atom. Two types of samples have been irradiated at incidence angles of 0 and 60 degree respectively. IBA was performed in-situ between the bombardment sessions with $2.5 \mathrm{MeV}{ }^{3} \mathrm{He}$ ions to record the evolution of the carbon fraction in the surface with increasing fluence. The amount of implanted $\mathrm{C}$ was measured using the nuclear reaction ${ }^{12} \mathrm{C}\left({ }^{3} \mathrm{He}, \mathrm{p}\right){ }^{14} \mathrm{~N}$ [11] with an experimental error below $10 \%$. Additionally, the application of thin $\mathrm{W}$ layers allows one to use Rutherford back-scattering (RBS) to record the evolution of the W areal density with an accuracy of $1 \%$. The main error source of this measurement is usually the uncertainty of the tabulated stopping power, which in this case cancels out because the change of the $\mathrm{W}$ areal density is measured relative to the initial value. Further details of the experimental setup and the measurement techniques are described in [10].

W layers with a thickness of $280 \pm 30 \mathrm{~nm}$ were prepared by magnetron sputter deposition both onto $\mathrm{Si}$ and onto polished $\mathrm{C}$ substrates with an intermediate $\mathrm{Cu}$ layer of $380 \pm 40 \mathrm{~nm}$. The thickness of the $\mathrm{W}$ layer was chosen to be always large enough to prevent interaction of projectiles with the $\mathrm{Cu}$ inter-layer and the substrate. Further details on structure and properties 
of the W layers can be found in [12]. All samples were produced in the same deposition process. Figure 6 shows images of the W surface for layers deposited on Si and C substrate respectively obtained by secondaryscanning electron microscopy (SEM). The latter sample shows a much higher roughness level with a typical height of 100-300 nm. Samples with polished Si substrate show a much lower typical roughness with an average height of $\approx 30$ $50 \mathrm{~nm}$.

\section{Results and discussion}

\subsection{Evolution of surface composition}

The evolution of the surface composition of the two types of samples, bombarded at $\alpha=0^{\circ}$, are shown in Figure 1a. Both types of samples show the same characteristic behaviour with regard to W sputtering and increase of implanted C. At small fluences $<2 \times 10^{17} \mathrm{~cm}^{-2}$, incident $\mathrm{C}$ ions arrive still at a nearly pure $\mathrm{W}$ surface resulting in a high implantation rate, which is also similar for both types of surfaces. By further sputtering of $\mathrm{W}$ finally the layer of implanted $\mathrm{C}$ becomes exposed leading to interaction of $\mathrm{C}$ ions with a mixed $\mathrm{W}+\mathrm{C}$ surface. At even higher fluence, the continuous accumulation of $\mathrm{C}$ in the surface leads to the formation of a closed pure $\mathrm{C}$ layer, which continues to grow proportional to the incident $\mathrm{C}$ flux. Smooth W surfaces of samples with Si substrate show a faster $\mathrm{C}$ accumulation than the rougher $\mathrm{W}$ surface of layers deposited on polished $\mathrm{C}$ substrate. Consequently, one can infer that roughness leads to an increased W sputter yield and to a slower accumulation of implanted C, with consequently delayed formation of a closed $\mathrm{C}$ layer.

Comparison of the experimental data to simulations with the Monte-Carlo code TRIDYN shows good quantitative agreement in case of the smoother surface at incidence angle $\alpha=0^{\circ}$, while the measurements for rough surfaces significantly deviate from the simulations. The good agreement for smooth surfaces indicates that the binary collision 
approximation in TRIDYN is an adequate model for the implantation and sputtering processes and that the influence of chemical effects, like carbide formation, can be neglected. The deviation in the case of rough surfaces must therefore be explained as an effect of the changed surface morphology only, since both samples were produced in the same process and the mechanical and chemical properties of the layers are similar. An approach to describe the sputtering dynamics for non-flat surface topography, developed by Kuestner et.al., is based on replacing the single incidence angle at flat surfaces by their distribution function [13], [14]. In addition, for rough surfaces this model takes into account local re-deposition of atoms emerging from the surface. The authors have shown that the re-deposition effect becomes an important factor only for very rough surfaces with steep slopes and can generally be neglected for smoother topographies, like those in the experiments discussed here. However, this work has been performed only for volatile projectiles and therefore the modification of the sample composition was neglected.

A simplification of this approach is necessary for TRIDYN simulations including the changing surface composition because the code can only simulate a $1 \mathrm{D}$ system with variations of the depth scale only. Therefore, the angle of incidence distribution is approximated by a mean angle of incidence as input parameter for the TRIDYN code, and re-deposition is neglected. Although such a strong simplification allows only qualitative comparisons with the experiment, it is for the presently available TRIDYN version the only way to take into account contributions of the evolution of the surface composition. Moreover, the growth of the $\mathrm{C}$ layer strongly affects the surface topography (see subsection 3.4), leading to a continuous change of the incidence angle distribution during the bombardment. Therefore, the simplified approach can be valid only for relatively low fluences. In case of the rougher surface, TRIDYN simulations assuming a mean incidence angle of $\alpha=38^{\circ}$ can qualitatively reproduce the composition dynamics of the sample surface. 
Figure $1 \mathrm{~b}$ shows that irradiation at $\alpha=60^{\circ}$ results in much faster removal of $\mathrm{W}$ from the surface, while the amount of implanted $C$ stays constant at fluences $>1.5 \times 10^{17} \mathrm{~cm}^{-2}$. After reaching equilibrium, $\mathrm{W}$ atoms are sputtered continuously from a mixed $\mathrm{W}+\mathrm{C}$ surface. It should be noted that a rough surface is still changing its topography in this fluence range and total stationary conditions including topography will be reached only at much higher fluences. Smooth surfaces, however, keep their flat topography (see subsection 3.4). Still, the dynamics of surface modification for different initial surface topography are nearly identical, and both can be reproduced best by TRIDYN assuming an effective incidence angle of $\alpha=70^{\circ}$. The shift of the best fitting mean incidence angle relative to the nominal value indicates that even the slight deviations from flat topography of the smooth surface are sufficient to cause an increased sputter yield and a decreased amount of $\mathrm{C}$ implanted after reaching equilibrium. On the other hand, samples with different surface roughness show nearly the same dynamics of surface composition evolution and, thus, one can apply the same effective incidence angle for an adequate simulation. The shift of data points due to $\mathrm{W}$ sputtering, measured for smooth surfaces, could be a result of the initial error in the fluence measurement or result from partial shadowing of the incident ions by the rough surface. The key parameter is the slope of the data points, which defines the sputter yield and which is the same for both types of surfaces.

\subsection{Implantation-sputtering curves}

One of the main difficulties of weight-loss measurements in this kind of experiments is the non-uniformity of the incident ion flux over the bombarded area in cases, where formation and growth of a protective layer is possible. A non-uniform lateral distribution of the incident flux results in a large experimental error in determining the dynamics of erosion and implantation by weight change measurements, because at edge areas of the irradiation spot exposed to lower beam intensities, sputtering might still dominate resulting in a corresponding decrease of the sample weight, while the central area with higher beam 
intensity will already have turned to a growing layer, resulting in an increase of the sample weight. Ion beam analysis allows local measurements of the surface composition and, at the same time, to monitor the lateral uniformity of the local flux by the shape of partial W RBS spectrum., The separate measurement of both elements allows one to exclude any explicit dependence on fluence and related inaccuracies. Since the change of the areal densities of C and W atoms $N_{C}$ and $N_{W}$ in the surface are functions of the incident fluence $\Phi$, the change of surface composition can be expressed as a parametric dependence:

$$
\left\{\begin{array}{l}
N_{W}=N_{W}(\Phi) \\
N_{C}=N_{C}(\Phi)
\end{array}\right.
$$

Such a parametric representation of experimental and simulation results is shown in Figure 2. The evolution of the surface composition under ion bombardment, expressed by an implantation-sputtering curve, depends only on properties of the surface and projectile atoms, and the kinematic parameters of the incident ions. The implantation-sputtering curve shows directly the relation between the amount of implanted projectiles and the amount of sputtered target atoms. The only uncertainty of this approach is the uniform bombardment of the surface area analysed by IBA. This can be verified by the slope of the low energy edge of the W partial spectrum, which would change with incident fluence in case of non-uniform W sputtering and $\mathrm{C}$ implantation.

Figure 2a shows an increase of the $\mathrm{C}$ areal density while $\mathrm{W}$ is not sputtered any more. This indicates that the surface is already fully covered with a protective $\mathrm{C}$ layer. The sample with higher roughness level is eroded much stronger before a protective $\mathrm{C}$ layer is formed and W sputtering begins to decrease. The difference between the experimental results for smooth and rough surface morphology can not be explained by experimental inaccuracies, like surface contamination, non-uniform beam profile, etc. The only difference in the two ion beam irradiation experiments is the roughness level. TRIDYN simulations with $\alpha=0^{\circ}$ 
accurately reproduce the experimental data for a smooth surface. For the data of the rough surface, simulations assuming an effective mean incidence angle of $\alpha=38^{\circ}$ show a good qualitative agreement with the data. However, on has to take into account that the topography modification by the growth of a C layer (see subsection 3.4) is not included in the code yet. The experimental data and simulations show consistently that the influence of roughness is leading to an increased sputter yield and a simultaneously decreased implantation rate. Both effects originate from the change of the distribution of local incidence angles towards higher values for an increasing fraction of the surface. This leads to a corresponding increase of a fraction of local incidence angles to values above the average incidence angle. This results in a higher sputter yield of implanted $\mathrm{C}$ and a higher $\mathrm{C}$ reflection coefficient.

The implantation-sputtering curve for the experiment with $\alpha=60^{\circ}$ in Figure $2 \mathrm{~b}$ shows that a factor of two less implanted carbon is sufficient to achieve equilibrium in comparison to TRIDYN data obtained for the same angle. Although the simulation with an effective mean incidence angle $\alpha=70^{\circ}$ shows better qualitative agreement, it does not, as mentioned in subsection 3.1, reflect the actual processes of topography modification.

\subsection{Elemental depth profiling}

The shape of the high energy edge of the tungsten partial RBS spectrum is determined by the concentration depth profile of implanted carbon. RBS analysis of an irradiated sample with a W layer on Si has shown that this part of spectrum has a characteristic shape in the fluence range of $(1.3-1.6) \times 10^{18} \mathrm{~cm}^{-2}$. It appears as a small peak at channels $\approx 1970-1990$ reflecting the depth distribution of implanted $\mathrm{C}$ near the surface (see Figure 3). After $\mathrm{C}$ implantation of a rough $\mathrm{W}$ surface, however, this characteristic feature does not occur even at highest fluences because of the averaging effect of the roughness on the $\mathrm{C}$ depth profile. To extract elemental depth profiles, the RBS spectrum was deconvoluted with the use of Bayesian statistical analysis $[15,16]$. Since differences of surface roughness result in 
qualitatively different spectral shapes, only spectra of smooth surfaces were deconvoluted. Generally, the result of the deconvolution procedure can be considered as a realistic depth profile only in cases, where the typical concentration profile depth scale is much higher than the elevation scale of the rough surface topography. For the samples of this study, the two scales are comparable, and therefore the depth profile for the rough surfaces will have a considerable error.

Comparison of $\mathrm{C}$ depth profiles obtained by the deconvolution procedure with profiles calculated by TRIDYN are presented in Figure 4. Dashed lines denote the error range of the Bayesian deconvolution, however without experimental uncertainties. The simulation and the deconvolution of the experimental RBS spectrum agree very well, particularly considering the influence of roughness and the uncertainties of stopping power and the unknown density of the mixed W-C layer. The influence of the last two factors becomes obvious comparing the depth profiles beyond an irradiation fluence of $1.6 \times 10^{18} \mathrm{~cm}^{-2}$. Although the relative amount of the $\mathrm{C}$ concentration is reproduced well, the thickness of the $\mathrm{C}$ layer then has an error of $\approx 10 \%$. After bombardment, the sample with Si substrate was broken apart along a crystal plane such that the crack ran across the irradiated spot. The obtained cross-section of the sample is shown in Figure 5. The image was taken by a SEM using back-scattered electrons in order to distinguish layers of different elements. The thickness of the grown $\mathrm{C}$ layer at the surface after an incident fluence of $1.6 \times 10^{18} \mathrm{~cm}^{-2}$ is $\approx 80-95 \mathrm{~nm}$, which agrees well with the results of both the RBS deconvolution and the TRIDYN simulations within the experimental and modelling uncertainties. The accuracy of the SEM measurement is limited by the microscope resolution, while the errors of both RBS analysis and TRIDYN simulation originate from the assumption of a linear superposition of the densities of pure $\mathrm{C}$ and $\mathrm{W}$ in the mixed material. Vice versa, one can infer from the observed agreement that the $\mathrm{C}$ density in the grown $\mathrm{C}$ layer is close to the density of pure graphite $\left(1.136 \times 10^{23} \mathrm{~cm}^{-3}\right)$, as assumed in the simulations. 


\subsection{Evolution of surface topography}

The surface topography of the samples has been studied by SEM. Before bombardment, the surface relief differs significantly by scale and particularly by altitude for the two substrates (Figure 6). Nuclear reaction analysis has shown that the surface is completely covered by the $\mathrm{W}$ layer without open pores extending to the substrate. With increasing fluence, the smooth surface samples keep their relief structure, while the average altitude of the rougher surface is decreasing (Figure 7). This is caused by both sputtering of surface peaks and by the growth of the $\mathrm{C}$ layer in the valleys. The image in Figure 7 was taken in the edge region of the irradiated spot, where the ion beam flux decreases, and therefore allows one to estimate the evolution of the surface topography as function of fluence.

The bombardment was stopped after the RBS spectra showed that no W sputtering was detectable any more, i.e. when a closed $\mathrm{C}$ layer had been formed to protect the $\mathrm{W}$ surface from further sputtering. Figure 8 shows the topography of the surface $\mathrm{C}$ layer, which appears similar on both types of samples with a generally smoother surface than prior bombardment. This shows that the growth of the $\mathrm{C}$ layer is accompanied by a flattening of the surface topography. At inclined incidence angle, $\alpha=60^{\circ}$, the surface flattening was not observed below a fluence of $2 \times 10^{17} \mathrm{~cm}^{-2}$ (Figure 9). At the same time, the topography of the smoother surface did not change significantly, while the rougher surfaces tend to decrease their altitude due to sputtering.

\section{Conclusions}

W layers with different levels of surface roughness were irradiated with $6 \mathrm{keV} \mathrm{C}$ ions at incidence angles of $\alpha=0^{\circ}$ and $\alpha=60^{\circ}$. IBA has been used to provide qualitatively new experimental data on sputtering and implantation processes with more detail than weight-loss measurements. The method eliminates errors originating from the fluence measurement by 
parametric data representation in the form of sputtering-implantation curves. It could be shown that the dynamics of the evolution of the surface composition can be comprehensively described by a kinematic binary collision model, like that implemented in the Monte-Carlo code TRIDYN. However, deviations of the surface topography from ideal planar geometry introduce several new factors: the local incidence angle for each projectile may strongly differ from the average beam incidence angle, while sputtering and implantation dynamically change the surface topography. At the same time, the growth of a $\mathrm{C}$ layer is accompanied by a flattening of the surface, which allows one to predict accurately the growth rate after reaching equilibrium. In comparison to the observed effects, contributions of chemical processes to sputtering and implantation can be neglected.

The observed influence of roughness on the dynamics of $\mathrm{C}$ implantation has an important consequence for simultaneous bombardment of $\mathrm{W}$ surfaces with $\mathrm{C}$ and $\mathrm{D}$ ions. Roughness would decrease the amount of $\mathrm{C}$ implanted by stronger sputtering and reflection, which can be misinterpreted as chemical sputtering due to formation of $\mathrm{C}-\mathrm{H}$ bonds. It would also shift the transition from continuous $\mathrm{W}$ sputtering to growth of $\mathrm{C}$ layers towards higher $\mathrm{C}$ fractions in the incident flux. While chemical sputtering should remain constant with permanent $\mathrm{C}$ concentration at the surface, increased sputtering due to roughness depends on a particular surface topography and may vary from one experiment to another.

\section{Acknowledgements}

The authors would like to thank Drs. Ch. Linsmeier, M. Mayer for their suggestions and helpful discussions. We would also like to thank Dipl.-Ing. B. Plöckl for the engineering support and P. Matern, G. Matern, S. Lindig, M. Fusseder and J. Dorner for their valuable technical support. 


\section{References}

[1] G. Janeschitz, ITER JCT and ITER HTs, J. Nucl. Mat. vol. 290-293 p.1 (2001).

[2] D. Meade, et al., Mission and design of the fusion ignition research experiment, in: Proceedings of the 18th IAEA Conf. on Fusion Energy, Sorrento, Italy October, 2000, (CD-ROM), pp. IAEA-CN-77/FTP2/16, IAEA, Vienna, 2001.

[3] S. Nishio, et al., Conceptional design of advanced steady-state tokamak reactor, in: Proceedings of the 18th Conf. on Fusion Energy, Sorrento, Italy October, 2000, (CDROM), pp. IAEA-CN-77/FTP2/14, IAEA, Vienna, 2001.

[4] K. Krieger et al., J. Nucl. Mat. Vol.266 -269 p.207 (1999).

[5] W.Eckstein, J.Roth, Nucl. Instrum. and Methods in Phys. Res., vol.B83, p.279 (1991).

[6] W. Moeller, W. Eckstein, J.P. Biersack, Comput. Phys. Commun. Vol.51 p.355 (1988).

[7] W. Eckstein, K. Krieger, J. Roth, J. Nucl. Mat. Vol.258-263 p.912-916 (1998).

[8] K.Schmid, J.Roth, J. of Nucl. Mat. vol.313 -316 p.302 -310 (2003).

[9] K. Schmid, J. Roth. J. of Nucl. Mat. Vol. 302 p.96-103 (2002).

[10] I. Bizyukov, K. Krieger, Rev. Sci. Instr, vol 77, 043501 (2006).

[11] S.Y. Tong, W.N. Lennard, P.F.A. Alkemada, I.V. Mitchell, Nucl. Instrum. Methods vol. B45, p.41, (1990)

[12] I. Bizyukov, K. Krieger, N. Azarenkov, S. Levchuk, Ch. Linsmeier, J. Nucl. Mat. vol.337-339 p.965 (2005).

[13] M. Küstner, W. Eckstein, V. Dose, J. Roth. Nucl. Instrum. and Methods in Phys. Res., vol.B145, p.320 (1998). 
[14] M. Küstner, W. Eckstein, E. Hechtl, J. Roth. J. Nucl. Mat. Vol. 265 p.22-27 (1999).

[15] U. Toussaint, R. Fischer, K. Krieger, V. Dose, New J. Phys. 1 (1999) 11.1

[16] U. v. Toussaint, V. Dose, Appl. Phys. A 82, 403-413 (2006) 


\section{List of figure captions}

Figure 1. Comparison of experimental and simulated surface composition vs. fluence. $\mathrm{W}$ is bombarded by $6 \mathrm{keV} \mathrm{C}$ projectiles at incidence angle of $\alpha=0^{\circ}$ (a) and $\alpha=60^{\circ}$ (b).

Figure 2. Representation of experimental and simulated by implantation-sputtering curves. $\mathrm{W}$ is bombarded by $6 \mathrm{keV} \mathrm{C}$ projectiles at incidence angle of $\alpha=0^{\circ}$ (a) and $\alpha=60^{\circ}$ (b).

Figure 3. Partial tungsten RBS spectra for smooth and rough surfaces (Si substrate and pyrolithic graphite substrate respectively) influenced by $\mathrm{C}$ implantation after a fluence of $1.5 \times 10^{18} \mathrm{~cm}^{-2}$.

Figure 4. Evolution of the $\mathrm{C}$ depth distribution with fluence. Triangles correspond to simulation of profiles with TRIDYN; solid line represents Bayesian deconvolution of measured spectra; dashed line denotes the error range of the deconvolution.

Figure 5. Image of cross-section of the implanted sample after cracking obtained by SEM in back-scattering electrons.

Figure 6. Topography of smooth (W on $\mathrm{Si}$ ) (a) and rough (W on $\mathrm{C}$ ) (b) surface before bombardment.

Figure 7. Topography of smooth (W on $\mathrm{Si}$ ) (a) and rough (W on $\mathrm{C}$ ) (b) surface after fluence of $\approx 10^{18} \mathrm{~cm}^{-2}, \alpha=0^{\circ}$

Figure 8. Topography of smooth (W on $\mathrm{Si}$ ) (a) and rough (W on C) (b) surface covered with grown $\mathrm{C}$ layer, $\alpha=0^{\circ}$

Figure 9. Topography of smooth (W on $\mathrm{Si}$ ) (a) and rough (W on $\mathrm{C}$ ) (b) surface after fluence $\approx 2 \times 10^{17} \mathrm{~cm}^{-2}, \alpha=60^{\circ}$ 

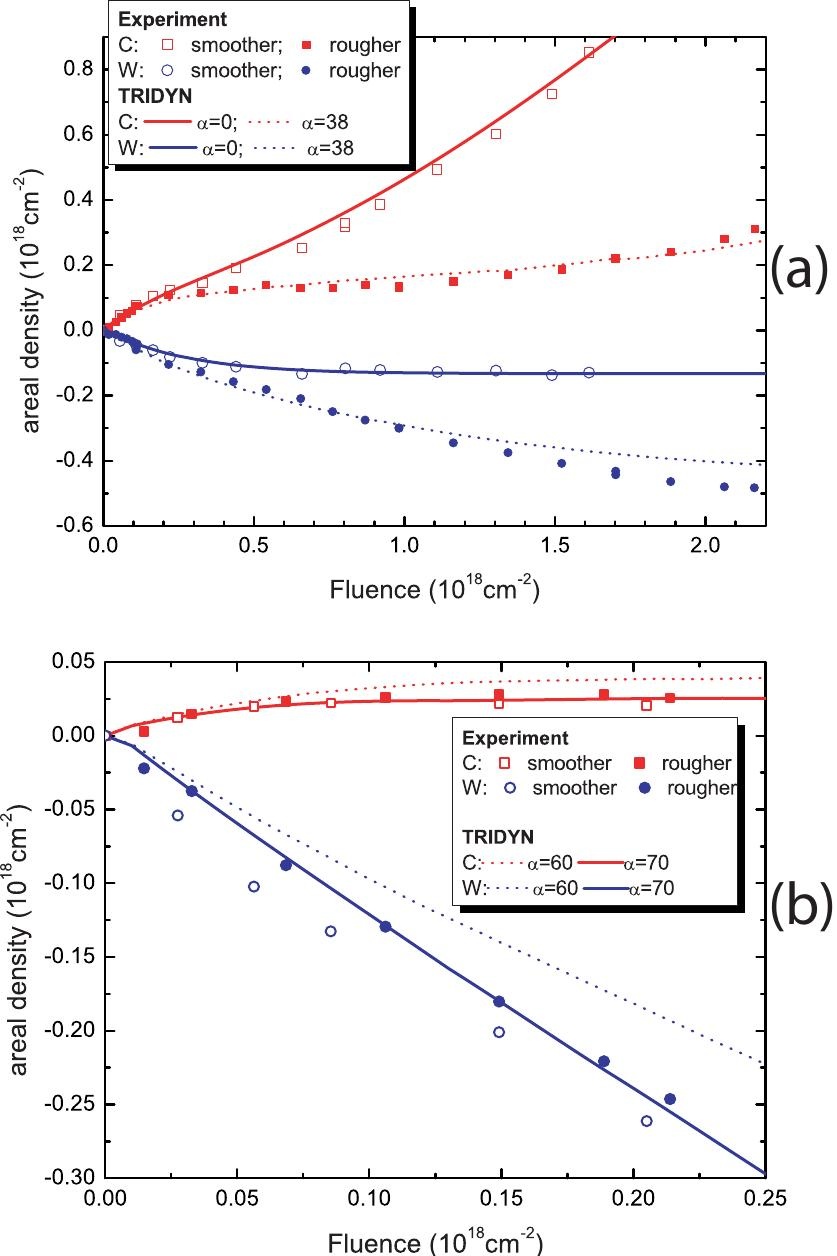

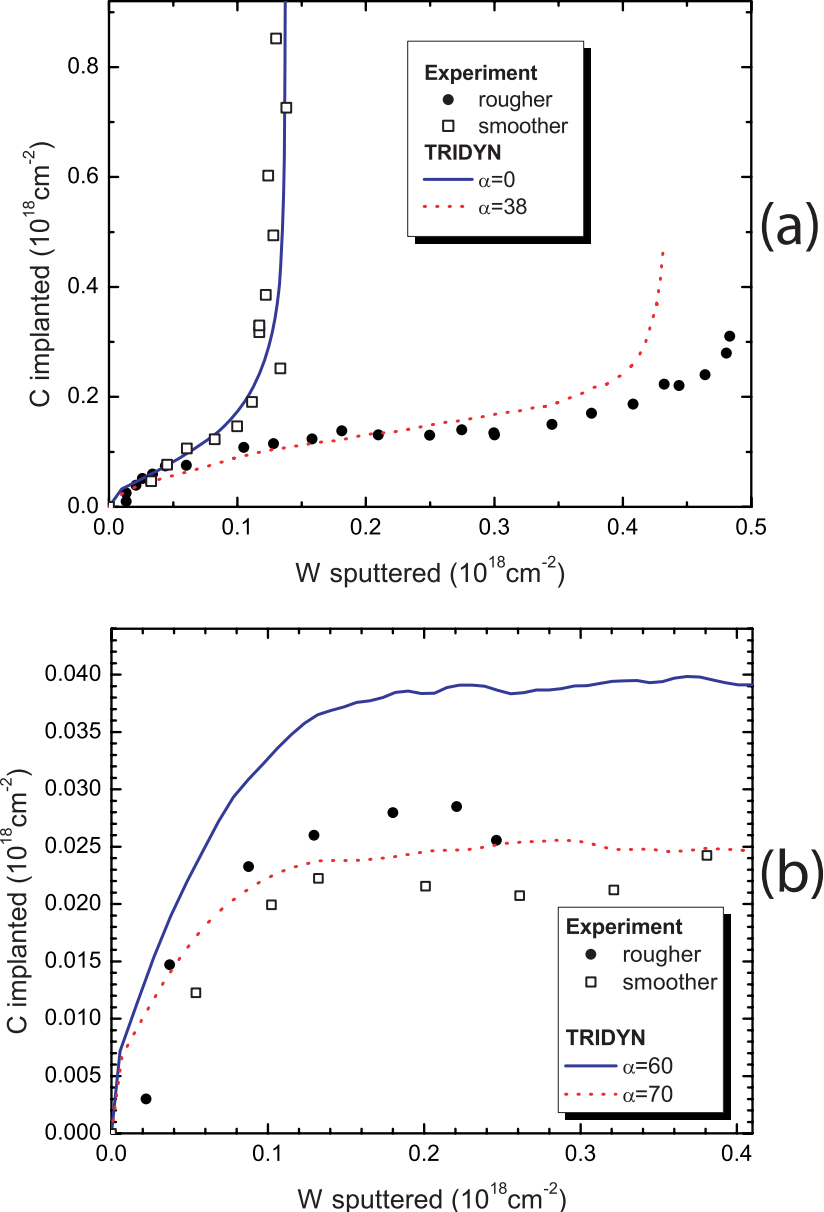


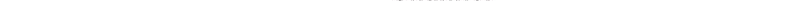





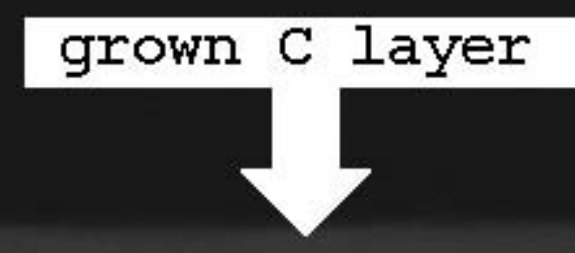

\section{w layer}

\section{$\mathrm{Cu}$ layer}

Si substrate

Acc.V Spot Magn Det WD $30.0 \mathrm{kV} 1.150000 \mathrm{x}$ BSE 9.4 Cross section $W$ on Si $3.5 \mathrm{~mm}$ 


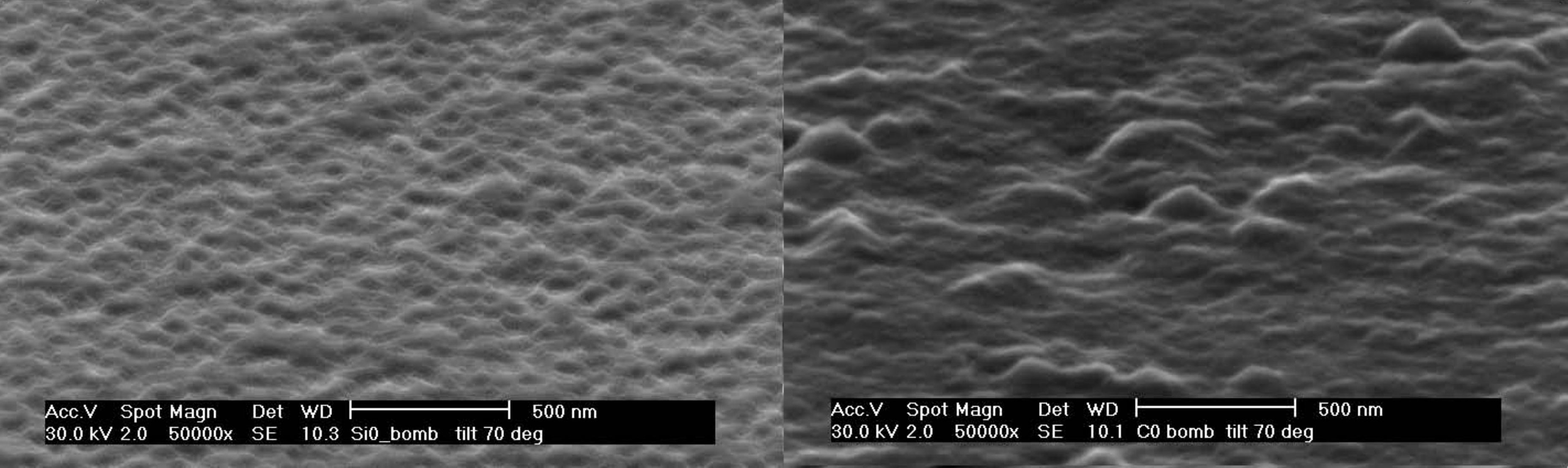


